\title{
Zugarzt in Russland und Afrika
}

\section{Tanja Kühnle}

Dipl.-Biol., Managing Editor

Die letzte Prüfung ist geschafft, das Medizinstudium beendet. Und wie geht es jetzt weiter? In unserer Serie «Du findest Deinen Weg!» stellen wir Ihnen in unregelmässigen Abständen aussergewöhnliche Berufsperspektiven für Mediziner vor. In dieser Ausgabe berichtet Dr. Jürgen Preimesberger von seiner Tätigkeit als Zugarzt.

Herr Preimesberger, wie kam es dazu, dass Sie Zugarzt geworden sind?

Das hat mit meiner Reiselust zu tun. Noch bis vor kurzem war ich mit über 130 Ländern der meistgereiste Österreicher. Die konkrete Idee kam dann durch einen Fernsehbericht über Rovos Rail in Afrika und den Zugarzt an Bord. Das fand ich spannend - also habe ich mich beworben.

\section{Brauchen Sie für diese Tätigkeit eine Zusatz- ausbildung?}

Nein. Ich habe jedoch nach meinem Facharzt eine Ausbildung zum Notfallmediziner gemacht. Zudem bin ich sechs Jahre lang auf verschiedenen Kreuzfahrtschiffen* als Arzt unterwegs gewesen. Ich bin es also gewohnt, allein mit Notsituationen umzugehen.

\section{Würden Sie diesen Beruf auch jungen Ärzten empfehlen?}

Da man gänzlich auf sich gestellt ist, ist es ratsam, als erfahrener Arzt den Job zu machen.

\section{Wie oft waren Sie bisher als Zugarzt unterwegs?} Insgesamt dreimal. Zweimal mit der Transsibirischen Eisenbahn und einmal mit Rovos Rail in Afrika.

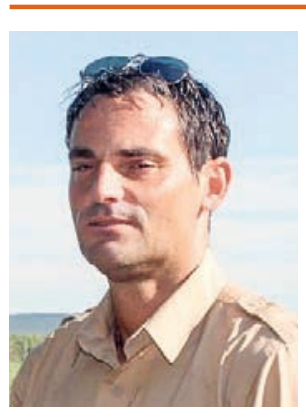

Name:

Dr. med. Jürgen Preimesberger Alter: 45

Zivilstand: in einer Partnerschaft lebend, zwei Kinder

Als Zugarzt tätig seit: 2013

Dauer der Einsätze:

max. 3 Wochen pro Jahr

In der übrigen Zeit tätig als:

selbstständiger Allgemeinmediziner

in Neumarkt am Wallersee/Salzburg Ausbildung: Facharzt für Allgemeinmedizin und acht zusätzliche Diplome (darunter u.a. Notarzt, Palliativmedizin und Wundmanagement)

\section{Wie lang hat Ihr Einsatz jeweils gedauert?}

Ich war immer für drei Wochen als Zugarzt unterwegs. Als selbständiger Allgemeinmediziner kann ich bei fünf Wochen Ferien im Jahr leider nicht mehr Zeit dafür erübrigen.

\section{Wie haben Sie sich mit den Gästen an Bord verständigt?}

Das Publikum war sehr international, deshalb meistens auf Englisch oder Deutsch.
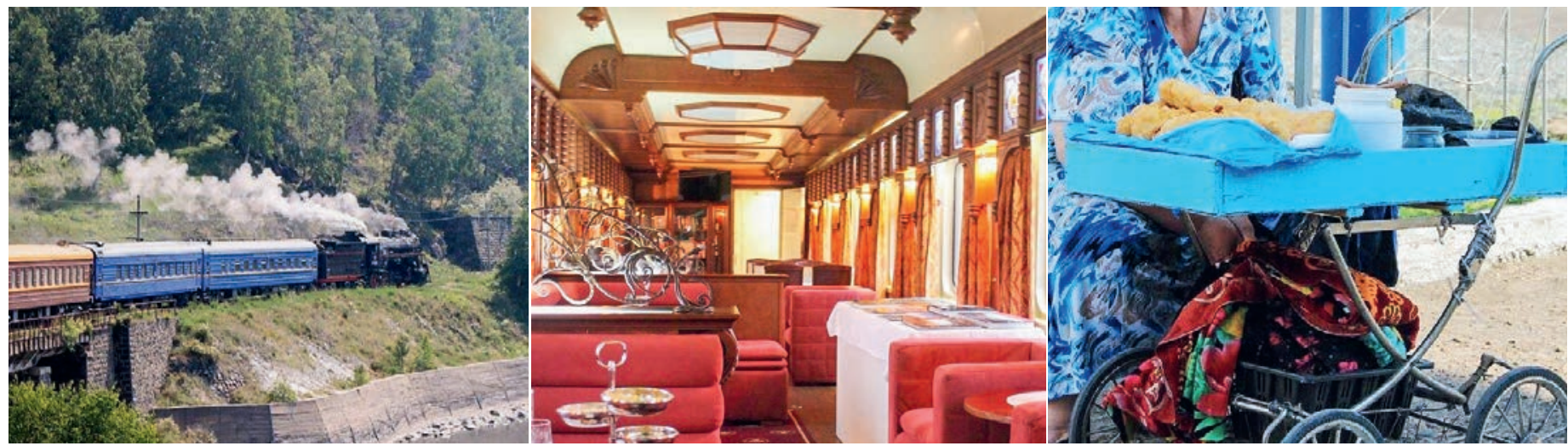

Die Transsibirische Eisenbahn ist mit 9288 km die längste Eisenbahnstrecke der Welt. Mehr als 400 Bahnhöfe liegen zwischen Moskau und Wladiwostok am Pazifik. An manchen Haltestellen bieten Einheimische auf kleinen Wagen diverse Köstlichkeiten zum Kauf an. 


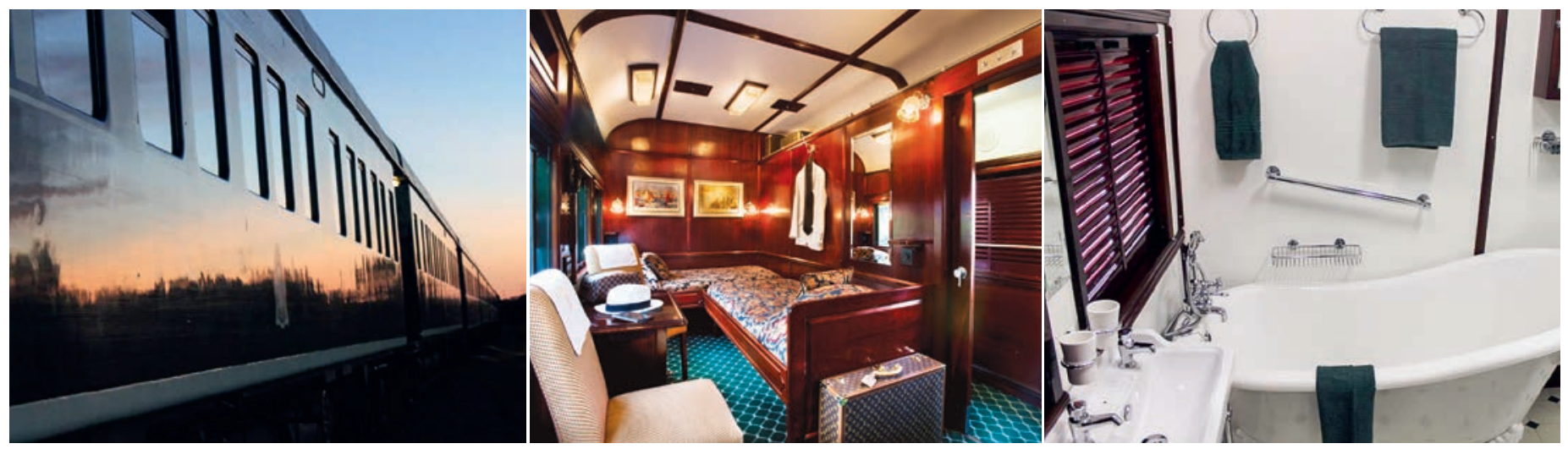

Rovos Rail bietet Luxuszugreisen durch Südafrika und darüber hinaus an. Beispielsweise werden regelmässig die Victoriafälle in Simbabwe angefahren. Auf den Fotos in der Mitte und rechts ist eine Kabine mit Bad zu sehen, wie sie auch dem Zugarzt zur Verfügung steht.

\section{Haben die Züge jeweils eine kleine Arztpraxis} an Bord?

Nein, der Zugarzt hat einen eigenen Waggon mit einer Kabine. Dort gibt es einen Koffer mit Medikamenten und Verbandsmaterial. Aber es ist kein kleines Hospital an Bord wie auf einem Kreuzfahrtschiff.

\section{Also gibt es keine Patientenliege, kein Labor und kein Ultraschall- oder Röntgengerät?}

Nein, es ist wirklich absolute Basic-Medizin.

Ich nehme an, es war auch keine Pflegefachperson mit dabei?

Nein, ich war allein.

\section{Welche Erkrankungen sind denn auf den Zugreisen typisch?}

Die meisten Reisenden haben Erkältungen oder grippale Infekte. Es war auch mal jemand mit einer Lungenentzündung dabei. Ansonsten ziehen sich die Passagiere manchmal auf Ausflügen Verstauchungen oder andere kleinere Verletzungen zu.

\section{Sind Sie auf den Ausflügen immer dabei?}

Ja, der Zugarzt muss an den Ausflügen teilnehmen.

\section{Hatte auch schon mal jemand eine ernste Erkrankung?}

Ja, ein Passagier hatte auf der Reise durch Afrika eine kardiale Dekompensation und einen kardialen Schock. Er hatte Glück im Unglück, denn wir waren gerade bei den Victoriafällen. Dort gibt es eine Krankenstation von International SOS, und wir konnten den Patienten nach Kapstadt ausfliegen, wo ihm geholfen werden konnte. Bei anderen Stationen wäre man, was die medizinische Hilfe betrifft, sehr limitiert gewesen.
Wäre ein Ausfliegen also nicht jederzeit möglich?

Nein, denn die Züge fahren in Sibirien wie auch in Afrika grösstenteils durch unbewohntes Gebiet. Manchmal hält man nur in kleinen Orten oder an einer Farm, die dann von den Gästen besichtigt werden kann. Man muss also im Einzelfall schauen, welche medizinische Versorgung vor Ort verfügbar ist und ob ein Helikopter organisiert werden kann.

\section{Hatte der Patient mit dem Herzinfarkt eine Vorerkrankung?}

Ja, er hatte einen Schrittmacher und eine ausgeprägte Herzinsuffizienz. Das war mir auch bekannt, denn die Reisenden müssen wie bei einer Kreuzfahrt einen Arztbrief mit den Erkrankungen und der aktuellen Medikation mitbringen. Im Falle eines Falles - wie hier - ist das sehr hilfreich. So konnte ich schnell reagieren, als ich bemerkt habe, dass es dem Passagier nicht gut geht.

Ist es schon mal vorgekommen, dass Sie einem Patienten nicht helfen konnten? Nein, zum Glück nicht.

\section{Haben Sie verglichen mit Ihrer Arztpraxis an Land etwas vermisst?}

Vermisst nicht. Ich habe nur mein eigenes Stethoskop und ein Pulsoximeter mitgenommen. Ausserdem mein eigenes Skriptum für die Medikamentendosierung.

\section{War unter den Passagieren schon einmal ein anderer Arzt?}

Nein, unter den Reisenden war während meiner Einsätze bisher noch kein anderer Arzt.

\section{Sind Sie selbst auch einmal krank geworden?} Nein, ich bin zum Glück nie krank geworden. 


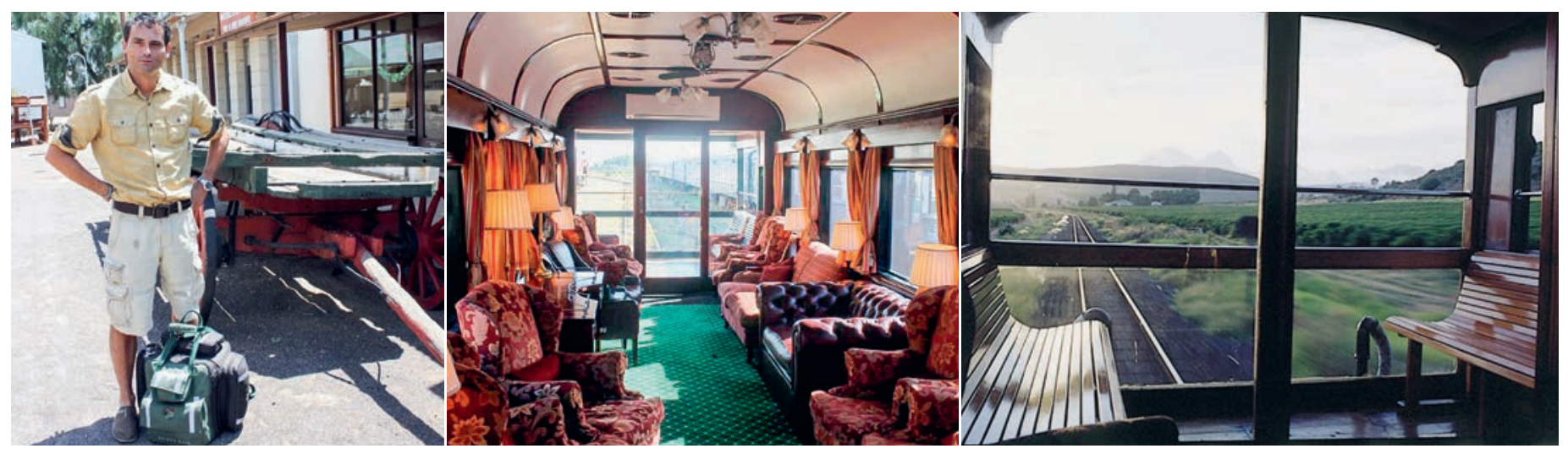

Jürgen Preimesberger (links) begleitet einen Ausflug in Südafrika. Immer dabei: der Rucksack mit seiner medizinischen Ausrüstung. Auf dem Foto in der Mitte ist der letzte Waggon mit der Aussichtsplattform zu sehen, der von den Passagieren gerne für Fotoshootings bei Tiersichtungen genutzt wird.

\section{Waren Sie als Zugarzt für die Reisenden rund um die Uhr verfügbar?}

Ja, man darf mich immer wecken, es gibt keine Sprechstunden. Die Passagiere wissen ohnehin schnell, wer ich bin. Schliesslich sind es nur rund 30 Reisende, und man verbringt viel Zeit miteinander, sei es auf den Ausflügen oder beim Essen im Speisewagen.

\section{Durfte Ihre Partnerin auf den Zugreisen dabei sein?}

Ja, der Partner darf mitfahren. Allerdings ist es nicht gratis wie auf dem Schiff, sondern es kostet 30 Prozent vom Reisepreis.

\section{Welche Zugreise gefällt Ihnen besser - die durch Transsibirien oder Südafrika?}

Das ist schwer zu sagen. Denn die beiden Reisen lassen sich eigentlich nicht miteinander vergleichen. Bei der Transsibirischen Eisenbahn ist der Hintergrund sehr interessant und auch die Tatsache, dass es die längste Eisenbahnstrecke der Welt ist. An Bord werden zudem tolle Vorträge gehalten, beispielsweise über Russland früher und heute.

Die Reise durch Afrika ist sicherlich aufgrund der Fauna und Flora und auch was den Zug an sich betrifft beeindruckender. Die Waggons sind im Stile der 1920er Jahre eingerichtet und sehr luxuriös. Am Zugende gibt es eine Plattform, auf der sich die Passagiere immer zum Fotoshooting treffen. Die Tierbeobachtungen, die Landschaft - das alles ist eine einzigartige Erfahrung.

\section{Gibt es eine andere Zugstrecke, die Sie gerne mal als Zugarzt begleiten würden?}

Ja, ich würde gerne durch Zentralasien fahren, nach Turkmenistan, Tadschikistan und Kasachstan. Aber ich habe noch nichts Konkretes geplant. Denn dieses Jahr bin ich wieder für drei Wochen als Schiffsarzt unterwegs.
* Anmerkung der Redaktion: Da wir den Beruf des Schiffsarztes bereits in einer früheren Ausgabe vorgestellt haben, konzentrieren wir uns in diesem Beitrag auf die Tätigkeit als Zugarzt. Wenn es Sie jedoch interessiert, welche Abenteuer Jürgen Preimesberger als Schiffsarzt erlebt hat, dann können Sie dies in seinem Buch Code Alpha nachlesen.

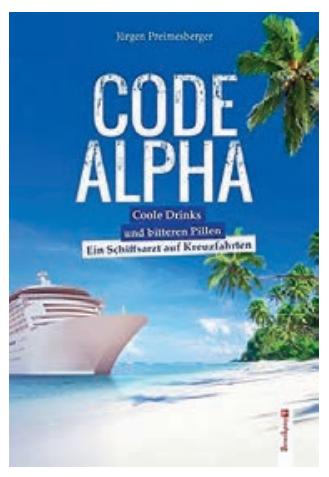

Jürgen Preimesberger CODE ALPHA: Coole Drinks und bittere Pillen Ein Schiffsarzt auf Kreuzfahrten

Wattens: Berenkamp-Verlag; 2017.

176 Seiten.

ISBN 978-3850933599

\section{Bildnachweis}

Foto auf S. 444 in der Mitte und 445 rechts: (C) Rovos Rail Alle anderen Fotos: Jürgen Preimesberger

\section{Bereits in dieser Serie erschienen: \\ - Rettungsarzt bei der Air Zermatt \\ Mannschaftsarzt beim FC Basel \\ - Gefängnisärztin \\ Expeditionsarzt \\ - Chief Medical Officer bei Sportverbänden Schiffsarzt auf Expeditionskreuzfahrten \\ Mit dem IKRK und MSF in Kriegsgebieten unterwegs Mediziner in der Forschung}

Haben Sie als Mediziner auch einen aussergewöhnlichen Beruf, den Sie unseren Lesern gern vorstellen möchten? Dann schicken Sie uns eine E-Mail an: tkuehnle[at]emh.ch 